\title{
A survey of s-unital and locally unital rings
}

\author{
PATRIK NYSTEDT* \\ University West, Department of Engineering Science, Trollhättan, Sweden.
}

\begin{abstract}
We gather some classical results and examples that show strict inclusion between the families of unital rings, rings with enough idempotents, rings with sets of local units, locally unital rings, s-unital rings and idempotent rings.
\end{abstract}

Keywords: Unital ring, rings with enough idempotents, rings with sets of local units, locally unital ring, s-unital ring, idempotent ring.

MSC2010: 1602, 16D99, 16S99, 16U99.

\section{Una revisión de anillos s-unitarios y localmente unitarios}

Resumen. Recopilamos algunos resultados clásicos y ejemplos que muestran una inclusión estricta entre las familias de anillos unitarios, anillos con suficientes idempotentes, anillos con conjuntos de unidades locales, anillos localmente unitarios, anillos s-unitarios y anillos idempotentes.

Palabras clave: Anillo unitario, anillos con suficientes idempotentes, anillos con conjuntos de unidades locales, anillo localmente unitario, anillo s-unitario, anillo idempotente.

\section{Introduction}

In many presentations of ring theory, authors make the assumption that all rings are unital, that is that they possess a multiplicative identity element. There are, however, lots of natural constructions in ring theory which share all properties of unital rings except the property of having a multiplicative identity. Such constructions include ideals, infinite direct sums of rings, and linear transformations of finite rank of an infinite dimensional vector space. For many examples of rings lacking a multiplicative identity there still exist weaker versions of identity elements. The purpose of the present article is to gather some classical results and examples of rings having different degrees of weak forms of identity

\footnotetext{
*E-mail: patrik.nystedt@hv.se

Received: 22 April 2019, Accepted: 23 May 2019.

To cite this article: P. Nystedt, A survey of s-unital and locally unital rings, Rev. Integr. temas mat. 37 (2019), No. 2, 251-260. doi: 10.18273/revint.v37n2-2019003.
} 
elements, ordered in hierarchy. To be more precise, we wish to show the following strict inclusions of families of rings:

$$
\begin{array}{rll}
\text { \{unital rings }\} & \subsetneq \text { \{rings with enough idempotents\} } \\
& \subsetneq \text { rings with sets of local units }\} \\
& \subsetneq \text { locally unital rings } \\
& \subsetneq \text { s-unital rings } \\
& \subsetneq \text { idempotent rings }\} \\
& \subsetneq \text { rings } .
\end{array}
$$

In our presentation, we will begin with the class of rings and narrow down our results and examples until we reach the class of unital rings.

\section{Idempotent and s-unital rings}

Definition 2.1. Throughout this article, $R$ denotes an associative ring. We do not assume that $R$ has a multiplicative identity. Let $\mathbb{Z}$ denote the set of integers and let $\mathbb{N}$ denote the set of positive integers.

Definition 2.2. The ring $R$ is called idempotent if $R^{2}=R$. Here $R^{2}$ denotes the set of all finite sums of elements of the form $r s$ for $r, s \in R$.

Example 2.3. It is easy to construct rings which are not idempotent. In fact, let $A$ be any non-zero abelian group. Define a multiplication on $A$ by saying that ab $=0$ for all $a, b \in A$. Then $A^{2}=\{0\} \neq A$.

Another generic class of examples is constructed in the following way. If $R$ is a ring and $I$ is a two-sided ideal of $R$, with $I^{2} \subsetneq I$, then $I$ is a ring which is not idempotent. This holds for many rings $R$, for instance when $R=\mathbb{Z}$ and $I$ is any non-trivial ideal of $R$.

The next definition was introduced by Tominaga in [10] and [11].

Definition 2.4. Let $M$ be a left (right) $R$-module. We say that $M$ is $s$-unital if for every $m \in M$ the relation $m \in R m(m \in m R)$ holds. If $M$ is an $R$-bimodule, then we say that $M$ is $s$-unital if it is $s$-unital both as a left $R$-module and as a right $R$-module. The ring $R$ is said to be left (right) $s$-unital if it is left (right) $s$-unital as a left (right) module over itself. The ring $R$ is said to be $s$-unital if it is $s$-unital as a bimodule over itself.

Example 2.5. The following example shows that there exist idempotent rings that are neither left nor right s-unital. Let $G=\{e, g\}$ denote the associative semigroup defined by the relations $e \cdot e=e$ and $e \cdot g=g \cdot e=g \cdot g=g$. Let $K$ denote a field and put $u=(1,0)$ and $v=(0,1)$ in $K \times K$. Let $R$ denote the twisted semigroup ring $(K \times K)[G]$ where the multiplication is defined by

$$
\left(x_{1}+x_{2} g\right)\left(y_{1}+y_{2} g\right)=x_{1} y_{1}+\left(x_{1} y_{2} e_{2}+x_{2} y_{1} e_{1}\right) g
$$

for $x_{1}, x_{2}, y_{1}, y_{2} \in K \times K$. Then $R$ is associative. Indeed, take

$$
x_{1}, x_{2}, y_{1}, y_{2}, z_{1}, z_{2} \in K \times K .
$$

[Revista Integración, temas de matemáticas 
A straightforward calculation shows that

$$
\left(\left(x_{1}+x_{2} g\right)\left(y_{1}+y_{2} g\right)\right)\left(z_{1}+z_{2} g\right)=x_{1} y_{1} z_{1}+\left(x_{2} y_{1} z_{1} e_{1}+x_{1} y_{1} z_{2} e_{2}\right) g
$$

and

$$
\left(x_{1}+x_{2} g\right)\left(\left(y_{1}+y_{2} g\right)\left(z_{1}+z_{2} g\right)\right)=x_{1} y_{1} z_{1}+\left(x_{2} y_{1} z_{1} e_{1}+x_{1} y_{1} z_{2} e_{2}\right) g .
$$

Also $R$ is neither left nor right s-unital. In fact, take $x_{1}, y_{2} \in K \times K$. If $g\left(x_{1}+x_{2} g\right)=g$, then $e_{1} x_{1} g=g$, so that $e_{1} x_{1}=(1,1)$ in $K \times K$ which is a contradiction. In the same way $\left(x_{1}+x_{2} g\right) g=g$ leads to $x_{1} e_{2}=(1,1)$ in $K \times K$ which is a contradiction. However, $R$ is idempotent since for all $(k, l) \in K \times K$, the following relations hold:

$$
(k, l) 1=(k, l) \cdot(1,1) \in R^{2},
$$

and

$$
(k, l) g=(k, 0) g \cdot(1,1) 1+(0, l) 1 \cdot(1,1) g \in R^{2} .
$$

Example 2.6. The following example (inspired by [7, Exercise 1.10]) shows that there are lots of examples of rings which are left (right) s-unital but not right (left) s-unital. Let $A$ be a unital ring with a non-zero multiplicative identity 1.

(a) Let $B_{l}$ denote the set $A \times A$ equipped with componentwise addition and multiplication defined by the relations

$$
(a, b)(c, d)=(a c, a d)
$$

for $a, b, c, d \in A$. Now we show that $B_{l}$ is associative. Take $a, b, c, d, e, f \in A$. Then,

$$
((a, b)(c, d))(e, f)=(a c, a d)(e, f)=(a c e, a c f),
$$

and

$$
(a, b)((c, d)(e, f))=(a, b)(c e, c f)=(a c e, a c f) .
$$

It is clear that any element of the form $(1, a)$, for $a \in A$, is a left identity for $B_{l}$. However, $B_{l}$ is not right unital. Indeed, since $(0,1) \notin\{(0,0)\}=(0,1) B_{l}$ it follows that $B_{l}$ is not even right s-unital. For each $n \in \mathbb{N}$ let $C_{n}$ denote a copy of $B_{l}$, and put $C=\oplus_{n \in \mathbb{N}} C_{n}$. Then $C$ is left s-unital but not left unital. Since none of the $C_{n}$ are right s-unital it follows that $C$ is not right s-unital.

(b) Let $B_{r}$ denote the set $A \times A$ equipped with componentwise addition and multiplication defined by the relation

$$
(a, b)(c, d)=(a c, b c)
$$

for $a, b, c, d \in A$. Now we show that $B_{r}$ is associative. Take $a, b, c, d, e, f \in A$. Then,

$$
((a, b)(c, d))(e, f)=(a c, b c)(e, f)=(a c e, b c e),
$$

and

$$
(a, b)((c, d)(e, f))=(a, b)(c e, d e)=(a c e, b c e) .
$$

It is clear that any element of the form $(1, a)$, for $a \in A$, is a right identity for $B_{l}$. However, $B_{r}$ is not left unital. Indeed, since $(0,1) \notin\{(0,0)\}=B_{r}(0,1)$ it follows that $B_{r}$ is not even left s-unital. For each $n \in \mathbb{N}$ let $D_{n}$ denote a copy of $B_{r}$ and put $D=\oplus_{n \in \mathbb{N}} D_{n}$. Then $D$ is right s-unital, but not right unital. Since none of the $D_{n}$ are left s-unital, it follows that $D$ is not left s-unital.

Vol. 37, $\left.\mathrm{N}^{\circ} 2,2019\right]$ 
Definition 2.7. If $e^{\prime}, e^{\prime \prime} \in R$, then put $e^{\prime} \vee e^{\prime \prime}=e^{\prime}+e^{\prime \prime}-e^{\prime} e^{\prime \prime}$.

Proposition 2.8. Let $M$ be a left (right) $R$-module. Then $M$ is left (right) s-unital if, and only if, for all $n \in \mathbb{N}$ and all $m_{1}, \ldots, m_{n} \in M$ there is $e \in R$ such that for all $i \in\{1, \ldots, n\}$ the relation $\mathrm{em}_{i}=m_{i}\left(m_{i} e=m_{i}\right)$ holds.

Proof. We follow the proof of [11, Theorem 1]. The "if" statements are trivial. Now we show the "only if" statements.

First, suppose that $M$ is a left $R$-module which is $s$-unital. Take $n \in \mathbb{N}$ and $m_{1}, \ldots, m_{n} \in$ $M$. Take $e_{n} \in R$ such that $e_{n} m_{n}=m_{n}$, and for every $i \in\{1, \ldots, n-1\}$ put $v_{i}=$ $m_{i}-e_{n} m_{i}$. By induction there is an element $e^{\prime} \in R$ such that for every $i \in\{1, \ldots, n-1\}$ the equality $e^{\prime} v_{i}=v_{i}$ holds. Put $e=e^{\prime} \vee e_{n}$. Then

$$
e m_{n}=e^{\prime} m_{n}+e_{n} m_{n}-e^{\prime} e_{n} m_{n}=e^{\prime} m_{n}+m_{n}-e^{\prime} m_{n}=m_{n},
$$

and for every $i \in\{1, \ldots, n-1\}$ we get that

$$
\begin{aligned}
e m_{i} & =e^{\prime} m_{i}+e_{n} m_{i}-e^{\prime} e_{n} m_{i} \\
& =e^{\prime}\left(m_{i}-e_{n} m_{i}\right)+e_{n} m_{i} \\
& =e^{\prime} v_{i}+e_{n} m_{i} \\
& =v_{i}+e_{n} m_{i} \\
& =m_{i}-e_{n} m_{i}+e_{n} m_{i} \\
& =m_{i} .
\end{aligned}
$$

Now suppose that $M$ is a right $R$-module which is $s$-unital. Take $n \in \mathbb{N}$ and $m_{1}, \ldots, m_{n} \in$ $M$. Take $e_{n} \in R$ such that $m_{n} e_{n}=m_{n}$, and for every $i \in\{1, \ldots, n-1\}$ put $v_{i}=$ $m_{i}-m_{i} e_{n}$. By induction there is an element $e^{\prime} \in R$ such that for every $i \in\{1, \ldots, n-1\}$ the equality $v_{i} e^{\prime}=v_{i}$ holds. Put $e=e_{n} \vee e^{\prime}$. Then

$$
m_{n} e=m_{n} e^{\prime}+m_{n} e_{n}-m_{n} e_{n} e^{\prime}=m_{n} e^{\prime}+m_{n}-m_{n} e^{\prime}=m_{n},
$$

and for every $i \in\{1, \ldots, n-1\}$ we get that

$$
\begin{aligned}
m_{i} e & =m_{i} e^{\prime}+m_{i} e_{n}-m_{i} e_{n} e^{\prime} \\
& =\left(m_{i}-m_{i} e_{n}\right) e^{\prime}+m_{i} e_{n} \\
& =v_{i} e^{\prime}+m_{i} e_{n} \\
& =v_{i}+m_{i} e_{n} \\
& =m_{i}-m_{i} e_{n}+m_{i} e_{n} \\
& =m_{i} .
\end{aligned}
$$

Proposition 2.9. Let $M$ be an $R$-bimodule and suppose that $e^{\prime}, e^{\prime \prime} \in R$. Let $X$ be a subset of $M$ such that for all $m \in X$ the relations $e^{\prime} m=m e^{\prime \prime}=m$ hold. Then; for all $m \in X$ the following relations hold:

$$
\left(e^{\prime \prime} \vee e^{\prime}\right) m=m\left(e^{\prime \prime} \vee e^{\prime}\right)=m .
$$

[Revista Integración, temas de matemáticas 
Proof. This is essentially the proof of [9, Lemma 1]. Take $m \in X$. Then

$$
\left(e^{\prime \prime} \vee e^{\prime}\right) m=\left(e^{\prime}+e^{\prime \prime}-e^{\prime \prime} e^{\prime}\right) m=e^{\prime} m+e^{\prime \prime} m-e^{\prime \prime} e^{\prime} m=m+e^{\prime \prime} m-e^{\prime \prime} m=m,
$$

and

$$
m\left(e^{\prime \prime} \vee e^{\prime}\right)=m\left(e^{\prime}+e^{\prime \prime}-e^{\prime \prime} e^{\prime}\right)=m e^{\prime}+m e^{\prime \prime}-m e^{\prime \prime} e^{\prime}=m e^{\prime}+m-m e^{\prime}=m .
$$

Proposition 2.10. Let $M$ be an R-bimodule. Then $M$ is s-unital if, and only if, for all $n \in \mathbb{N}$ and all $m_{1}, \ldots, m_{n} \in M$ there is $e \in R$ such that for all $i \in\{1, \ldots, n\}$ the relation em $m_{i}=m_{i} e=m_{i}$ holds.

Proof. The "if" statement is trivial. Now we show the "only if" statement. Take $n \in \mathbb{N}$ and $m_{1}, \ldots, m_{n} \in M$. From Proposition 2.8 it follows that there are $e^{\prime}, e^{\prime \prime} \in R$ such that for all $i \in\{1, \ldots, n\}$ the relations $e^{\prime} m_{i}=m_{i} e^{\prime \prime}=m_{i}$ hold. The claim now follows from Proposition 2.9 if we put $e=e^{\prime \prime} \vee e^{\prime}$ and $X=\left\{m_{1}, \ldots, m_{n}\right\}$.

Proposition 2.11. The ring $R$ is left (right) s-unital if, and only if, for all $n \in \mathbb{N}$ and all $r_{1}, \ldots, r_{n} \in R$ there is $e \in R$ such that for all $i \in\{1, \ldots, n\}$ the relation er $_{i}=r_{i}$ $\left(r_{i} e=r_{i}\right)$ holds.

Proof. This follows from Proposition 2.8 .

Proposition 2.12. The ring $R$ is s-unital if, and only if, for all $n \in \mathbb{N}$ and all $r_{1}, \ldots, r_{n} \in$ $R$ there is $e \in R$ such that for all $i \in\{1, \ldots, n\}$ the relations $e r_{i}=r_{i} e=r_{i}$ hold.

Proof. This follows from Proposition 2.10.

Definition 2.13. An element $e \in R$ is called idempotent if $e^{2}=e$.

Definition 2.14. We say that $R$ is left (right) locally unital if for all $n \in \mathbf{N}$ and all $r_{1}, \ldots, r_{n} \in R$ there is an idempotent $e \in R$ such that for all $i \in\{1, \ldots, n\}$ the equality $e r_{i}=r_{i}\left(r_{i} e=r_{i}\right)$ holds. We say that $R$ is locally unital if it is both left locally unital and right locally unital.

Example 2.15. Let $R$ denote the ring of real valued continuous functions on the real line with compact support. Then $R$ is s-unital, but neither left nor right locally unital.

\section{Locally unital rings}

The next definition was introduced by Ánh and Márki in [4].

Definition 3.1. The ring $R$ is said to be locally unital if for all $n \in \mathbb{N}$ and all $r_{1}, \ldots, r_{n} \in R$ there is an idempotent $e \in R$ such that for all $i \in\{1, \ldots, n\}$ the equalities $e r_{i}=r_{i} e=r_{i}$ hold.

Proposition 3.2. Suppose that $e^{\prime}, e^{\prime \prime} \in R$ are idempotents, and put $e=e^{\prime \prime} \vee e^{\prime}$. Then $e^{2}=e+e^{\prime} e^{\prime \prime}-e^{\prime} e^{\prime \prime} e^{\prime}-e^{\prime \prime} e^{\prime} e^{\prime \prime}+e^{\prime \prime} e^{\prime} e^{\prime \prime} e^{\prime}$. If either of the following equalities hold, 
(i) $e^{\prime} e^{\prime \prime}=e^{\prime}$,

(ii) $e^{\prime} e^{\prime \prime}=e^{\prime \prime}$,

(iii) $e^{\prime \prime} e^{\prime}=e^{\prime \prime}$,

(iv) $e^{\prime \prime} e^{\prime}=e^{\prime}$,

(v) $e^{\prime} e^{\prime \prime}=e^{\prime \prime} e^{\prime}$,

then e is idempotent.

Proof. A straightforward calculation shows that

$$
\begin{aligned}
e^{2} & =\left(e^{\prime}+e^{\prime \prime}-e^{\prime \prime} e^{\prime}\right)^{2} \\
& =\left(e^{\prime}\right)^{2}+e^{\prime} e^{\prime \prime}-e^{\prime} e^{\prime \prime} e^{\prime}+e^{\prime \prime} e^{\prime}+\left(e^{\prime \prime}\right)^{2}-\left(e^{\prime \prime}\right)^{2} e^{\prime}-e^{\prime \prime}\left(e^{\prime}\right)^{2}-e^{\prime \prime} e^{\prime} e^{\prime \prime}+e^{\prime \prime} e^{\prime} e^{\prime \prime} e^{\prime} \\
& =e^{\prime}+e^{\prime} e^{\prime \prime}-e^{\prime} e^{\prime \prime} e^{\prime}+e^{\prime \prime} e^{\prime}+e^{\prime \prime}-e^{\prime \prime} e^{\prime}-e^{\prime \prime} e^{\prime}-e^{\prime \prime} e^{\prime} e^{\prime \prime}+e^{\prime \prime} e^{\prime} e^{\prime \prime} e^{\prime} \\
& =e+e^{\prime} e^{\prime \prime}-e^{\prime} e^{\prime \prime} e^{\prime}-e^{\prime \prime} e^{\prime} e^{\prime \prime}+e^{\prime \prime} e^{\prime} e^{\prime \prime} e^{\prime} .
\end{aligned}
$$

Now we show the last part. If (i) holds, then

$e^{\prime} e^{\prime \prime}-e^{\prime} e^{\prime \prime} e^{\prime}-e^{\prime \prime} e^{\prime} e^{\prime \prime}+e^{\prime \prime} e^{\prime} e^{\prime \prime} e^{\prime}=e^{\prime}-\left(e^{\prime}\right)^{2}-e^{\prime \prime} e^{\prime}+e^{\prime \prime}\left(e^{\prime}\right)^{2}=e^{\prime}-e^{\prime}-e^{\prime \prime} e^{\prime}+e^{\prime \prime} e^{\prime}=0$.

If (ii) holds, then

$$
e^{\prime} e^{\prime \prime}-e^{\prime} e^{\prime \prime} e^{\prime}-e^{\prime \prime} e^{\prime} e^{\prime \prime}+e^{\prime \prime} e^{\prime} e^{\prime \prime} e^{\prime}=e^{\prime \prime}-\left(e^{\prime \prime}\right)^{2}-\left(e^{\prime \prime}\right)^{2}+\left(e^{\prime \prime}\right)^{3}=e^{\prime \prime}-e^{\prime \prime}-e^{\prime \prime}+e^{\prime \prime}=0 .
$$

If (iii) holds, then

$$
e^{\prime} e^{\prime \prime}-e^{\prime} e^{\prime \prime} e^{\prime}-e^{\prime \prime} e^{\prime} e^{\prime \prime}+e^{\prime \prime} e^{\prime} e^{\prime \prime} e^{\prime}=e^{\prime} e^{\prime \prime}-e^{\prime} e^{\prime \prime}-\left(e^{\prime \prime}\right)^{2}+\left(e^{\prime \prime}\right)^{2}=-e^{\prime \prime}+e^{\prime \prime}=0 .
$$

If (iv) holds, then

$$
e^{\prime} e^{\prime \prime}-e^{\prime} e^{\prime \prime} e^{\prime}-e^{\prime \prime} e^{\prime} e^{\prime \prime}+e^{\prime \prime} e^{\prime} e^{\prime \prime} e^{\prime}=e^{\prime} e^{\prime \prime}-\left(e^{\prime}\right)^{2}-e^{\prime} e^{\prime \prime}+\left(e^{\prime}\right)^{2}=-e^{\prime}+e^{\prime}=0 .
$$

If (v) holds, then

$$
\begin{aligned}
e^{\prime} e^{\prime \prime}-e^{\prime} e^{\prime \prime} e^{\prime}-e^{\prime \prime} e^{\prime} e^{\prime \prime}+e^{\prime \prime} e^{\prime} e^{\prime \prime} e^{\prime} & =e^{\prime} e^{\prime \prime}-\left(e^{\prime}\right)^{2} e^{\prime \prime}-e^{\prime}\left(e^{\prime \prime}\right)^{2}+\left(e^{\prime}\right)^{2}\left(e^{\prime \prime}\right)^{2} \\
& =e^{\prime} e^{\prime \prime}-e^{\prime} e^{\prime \prime}-e^{\prime} e^{\prime \prime}+e^{\prime} e^{\prime \prime} \\
& =0
\end{aligned}
$$

Proposition 3.3. A ring is locally unital in the sense of Definition 2.14 if, and only if, it is locally unital in the sense Definition 3.1.

Proof. The "only if" statement is immediate. Now we show the "if" statement. We use the argument from the proof of [6, Proposition 1.10] (see also [4, Example 1]). Suppose that $R$ is a ring which is locally unital in the sense of Definition 2.14. Take $n \in \mathbb{N}$ and $r_{1}, \ldots, r_{n} \in R$. Since $R$ is right locally unital, there is an idempotent $e^{\prime} \in R$ such that for all $i \in\{1, \ldots, n\}$ the equality $r_{i} e^{\prime}=r_{i}$ holds. Since $R$ is left locally unital, there is an idempotent $e^{\prime \prime} \in R$ such that $e^{\prime \prime} e^{\prime}=e^{\prime}$, and for all $i \in\{1, \ldots, n\}$ the equality $e^{\prime \prime} r_{i}=r_{i}$ holds. Put $e=e^{\prime} \vee e^{\prime \prime}$. From Proposition 3.2 it follows that $e$ is idempotent. From Proposition 2.9, with $X=\left\{r_{1}, \ldots, r_{n}\right\}$, it follows that for all $i \in\{1, \ldots, n\}$ the equalities $e r_{i}=r_{i} e=r_{i}$ hold. So, $R$ is locally unital in the sense of Definition 3.1.

[Revista Integración, temas de matemáticas 


\section{Regular rings}

Definition 4.1. The ring $R$ is called regular if for every $r \in R$ there is $s \in R$ such that $r=r s r$.

The next proposition is [4, Example 1].

Proposition 4.2. Every regular ring is locally unital.

Proof. We proceed in almost the same way as in the proof of Proposition 2.8. Let $R$ be a regular ring. Take $n \in \mathbb{N}$ and $r_{1}, \ldots r_{n} \in R$. First we show that $R$ is left locally unital. By induction there is an idempotent $e_{1} \in R$ such that for all $i \in\{1, \ldots, n-1\}$ the equality $e_{1} r_{i}=r_{i}$ holds. Put $s=r_{n}-e_{1} r_{n}$. Since $R$ is regular, there is $t \in R$ such that $s=$ sts. Put $f=s t$. Then $f$ is idempotent and

$$
e_{1} f=e_{1} s t=e_{1}\left(r_{n}-e_{1} r_{n}\right) t=\left(e_{1} r_{n}-e_{1}^{2} r_{n}\right) t=\left(e_{1} r_{n}-e_{1} r_{n}\right) t=0 .
$$

Put $g=f-f e_{1}$. Then $e_{1} g=g e_{1}=0$ and

$$
g^{2}=f^{2}-f^{2} e_{1}-f e_{1} f+f e_{1} f e_{1}=f-f e_{1}=g .
$$

Let $e=e_{1}+g$. Then $e$ is an idempotent. Take $i \in\{1, \ldots, n-1\}$. Then,

$$
e r_{i}=\left(e_{1}+g\right) r_{i}=\left(e_{1}+g\right) e_{1} r_{i}=\left(e_{1}^{2}+g e_{1}\right) r_{i}=e_{1} r_{i}=r_{i}
$$

Finally,

$$
\begin{aligned}
e r_{n} & =\left(e_{1}+g\right) r_{n} \\
& =e_{1} r_{n}+g r_{n} \\
& =e_{1} r_{n}+\left(f-f e_{1}\right) r_{n} \\
& =e_{1} r_{n}+f r_{n}-f e_{1} r_{n} \\
& =e_{1} r_{n}+f s \\
& =e_{1} r_{n}+s t s \\
& =e_{1} r_{n}+s \\
& =e_{1} r_{n}+r_{n}-e_{1} r_{n} \\
& =r_{n} .
\end{aligned}
$$

Now we show that $R$ is right locally unital. By induction there is an idempotent $e_{1} \in R$ such that for all $i \in\{1, \ldots, n-1\}$ the equality $r_{i} e_{1}=r_{i}$ holds. Put $s=r_{n}-r_{n} e_{1}$. Since $R$ is regular, there is $t \in R$ such that $s=s t s$. Put $f=t s$. Then $f$ is idempotent, and

$$
f e_{1}=t s e_{1}=t\left(r_{n}-r_{n} e_{1}\right) e_{1}=t\left(r_{n} e_{1}-r_{n} e_{1}^{2}\right)=t\left(r_{n} e_{1}-r_{n} e_{1}\right)=0 .
$$

Put $g=f-e_{1} f$. Then $e_{1} g=g e_{1}=0$ and

$$
g^{2}=f^{2}-e_{1} f^{2}-f e_{1} f+e_{1} f e_{1} f=f-e_{1} f=g .
$$

Let $e=e_{1}+g$. Then $e$ is an idempotent. Take $i \in\{1, \ldots, n-1\}$. Then,

$$
r_{i} e=r_{i}\left(e_{1}+g\right)=r_{i} e_{1}\left(e_{1}+g\right)=r_{i}\left(e_{1}^{2}+e_{1} g\right)=r_{i} e_{1}=r_{i} .
$$

Vol. 37, $\mathrm{N}^{\circ}$ 2, 2019] 
Finally,

$$
\begin{aligned}
r_{n} e & =r_{n}\left(e_{1}+g\right) \\
& =r_{n} e_{1}+r_{n} g \\
& =r_{n} e_{1}+r_{n}\left(f-e_{1} f\right) \\
& =r_{n} e_{1}+r_{n} f-r_{n} e_{1} f \\
& =r_{n} e_{1}+s f \\
& =r_{n} e_{1}+s t s \\
& =r_{n} e_{1}+s \\
& =r_{n} e_{1}+r_{n}-r_{n} e_{1} \\
& =r_{n} .
\end{aligned}
$$

\section{Rings with sets of local units}

The next definition was introduced by Abrams in [2].

Definition 5.1. Suppose that $E$ is a set of commuting idempotents in $R$ which is closed under the operation $\vee$ from Definition 2.7. Then $E$ is called a set of local units for $R$ if for all $r \in R$ there is $e \in E$ such that $e r=r e=r$.

Remark 5.2. In [2, Definition 1.1] the condition that $E$ is closed under $\vee$ was not included. However, since this was intended (personal communication with G. Abrams) we chose to include it here.

Proposition 5.3. If $R$ has a set of local units $E$, then for all $n \in \mathbf{N}$ and all $r_{1}, \ldots, r_{n} \in R$ there is $e \in E$ such that for all $i \in\{1, \ldots, n\}$ the equalities er $_{i}=r_{i} e=r_{i}$ holds.

Proof. Take $n \in \mathbb{N}$ and $r_{1}, \ldots r_{n} \in R$. By induction there is $e_{1}, e_{2} \in E$ such $e_{2} r_{n}=$ $r_{n} e_{2}=r_{n}$, and for all $i \in\{1, \ldots, n-1\}$ the relations $e_{1} r_{i}=r_{i} e_{1}=r_{i}$ hold. Put $e=e_{1} \vee e_{2}$. Then, since $e_{1} e_{2}=e_{2} e_{1}$, we get that

$$
e r_{n}=e_{1} r_{n}+e_{2} r_{n}-e_{1} e_{2} r_{n}=e_{1} r_{n}+r_{n}-e_{1} r_{n}=r_{n}
$$

and

$$
r_{n} e=r_{n} e_{1}+r_{n} e_{2}-r_{n} e_{2} e_{1}=r_{n} e_{1}+r_{n}-r_{n} e_{1}=r_{n},
$$

and for all $i \in\{1, \ldots, n-1\}$ we get that

$$
e r_{i}=e_{1} r_{i}+e_{2} r_{i}-e_{2} e_{1} r_{i}=r_{i}+e_{2} r_{i}-e_{2} r_{i}=r_{i}
$$

and

$$
r_{i} e=r_{i} e_{1}+r_{i} e_{2}-r_{i} e_{1} e_{2}=r_{i}+r_{i} e_{2}-r_{i} e_{2}=r_{i} .
$$

Proposition 5.4. If a ring has a set of local units, then it is locally unital.

Proof. This follows from Proposition 5.3.

Example 5.5. According to [4, Example 1] there are regular rings that do not possess sets of local units in the sense of Definition 5.1.

Definition 5.6. If $e, f \in R$ are idempotent, then $e$ and $f$ are said to be orthogonal if $e f=f e=0$. 


\section{Rings with enough idempotents}

The following definition was introduced by Fuller in [5].

Definition 6.1. The ring $R$ is said to have enough idempotents in case there exists a set $\left\{e_{i}\right\}_{i \in I}$ of orthogonal idempotents in $R$ (called a complete set of idempotents for $R$ ) such that $R=\oplus_{i \in I} R e_{i}=\oplus_{i \in I} e_{i} R$.

Example 6.2. There exist rings which have sets of local units in the sense of Definition 5.1, but which does not have enough idempotents in the sense of Definition 6.1. To exemplify this we recall the construction from $[1$, Example 1.6]. Let $F$ denote the field with two elements, and let $R$ be the ring of all functions $f: \mathbb{N} \rightarrow F$. For each $n \in \mathbb{N}$ define $f_{n} \in R$ by $f_{n}(n)=1$, and $f_{n}(m)=0$, if $m \neq n$. For all finite subsets $S$ of $\mathbb{N}$, define $f_{S} \in R$ via $f_{S}=\sum_{n \in S} f_{n}$. Then $I=\left\{f_{S} \mid S\right.$ is a finite subset of $\left.\mathbb{N}\right\}$ is an ideal of $R$. Since $R$ is unital, Zorn's lemma implies the existence of a maximal proper ideal $M$ of $R$ with $I \subseteq M$. Since all elements in $R$, and hence also in $M$, are idempotent, it follows that $M$ is a ring with $E=M$ as a set of local units. Seeking a contradiction, suppose that $M$ has a complete set of idempotents $\left\{e_{j}\right\}_{j \in J}$. Since $I$, and hence $M$, contains all $f_{n}$, for $n \in \mathbb{N}$, it follows that $1_{R}=\sum_{j \in J} e_{j}$. Since $M$ is a proper ideal, we get that $1_{R} \notin M$, and thus it follows that $J$ is an infinite set. Choose any partition $J=K \cup L$, with $K \cap L=\emptyset$, and $K$ and $L$ infinite. Define $e_{K}=\sum_{k \in K} e_{k}$ and $e_{L}=\sum_{l \in L} e_{l}$. Since the $e_{j}$ are pairwise orthogonal, we get that $e_{K} e_{L}=0$. But $M$ is a maximal ideal of $R$. Therefore $M$ is a prime ideal of $R$, and thus $e_{K} \in M$ or $e_{L} \in M$. Suppose that $e_{K} \in M$. Since $\left\{e_{j}\right\}_{j \in J}$ is a complete set of idempotents, there must exist a finite set $J^{\prime}$ of $J$ with $e_{K}=\sum_{j \in J^{\prime}} e_{j}$ which is a contradiction. Analogously, the case when $e_{L} \in M$ leads to a contradiction. Therefore, $M$ is not a ring with enough idempotents.

Definition 6.3. If $M$ is a left (right) $R$-module, then $M$ is called left (right) unital if there is $e \in R$ such that for all $m \in M$ the relation $e m=m(m e=m)$ holds. In that case $e$ is said to be a left (right) identity for $M$. If $M$ is an $R$-bimodule, then $M$ is called unital if it is unital both as a left $R$-module and a right $R$-module. The ring $R$ is said to be left (right) unital if it is left (right) unital as a left (right) module over itself. The ring $R$ is called unital if it is unital as a bimodule over itself.

Example 6.4. The ring $B_{l}$ (or $B_{r}$ ) from Example 2.6 is a ring which is left (or right) unital, but not right (or left) unital.

Example 6.5. There are many classes of rings that are neither left nor right unital but still have enough idempotents. Here are some examples:

- infinite direct sums of unital rings;

- category rings where the category has infinitely many objects (see e.g. [8, Proposition 4]);

- Leavitt path algebras with infinitely many vertices (see e.g. [3, Lemma 1.2.12(iv)]).

Proposition 6.6. Let $M$ be an R-bimodule. Then $M$ is unital if, and only if, there is $e \in R$ such that for all $m \in M$ the relations $e m=m e=m$ hold.

Vol. 37, $\left.\mathrm{N}^{\circ} 2,2019\right]$ 
Proof. The "if" statement is trivial. The "only if" statement follows from Proposition 2.9 if we put $X=M$.

Proposition 6.7. The ring $R$ is unital if, and only if, there is $e \in R$ such that for all $r \in R$ the relations er $=r e=r$ hold.

Proof. This follows from Proposition 6.6 if we put $M=R$.

Remark 6.8. Proposition 6.7 can of course be proved directly in the following way. Let $e^{\prime}$ (or $\left.e^{\prime \prime}\right)$ be a left (or right) identity for $R$ as a left (or right) module over itself. Then $e^{\prime}=e^{\prime} e^{\prime \prime}=e^{\prime \prime}$.

We end the article with the following remark, which connects unitality and s-unitality.

Proposition 6.9. If $R$ is left (right) s-unital and right (left) unital, then $R$ is unital.

Proof. First suppose that $R$ is left $s$-unital and right unital. Let $f$ be a right identity of $R$ and take $r \in R$. From Proposition 2.8 it follows that there is $e \in R$ with $e r=r$ and ef $=f$. But since $f$ is a right identity of $R$ it follows that $e f=e$. Thus $e=f$ and hence $f r=e r=r$ so that $f$ is a left identity of $R$. Now suppose that $R$ is right $s$-unital and left unital. Let $f$ be a left identity of $R$ and take $r \in R$. From Proposition 2.8 it follows that there is $e \in R$ with $r e=r$ and $f e=f$. But since $f$ is a left identity of $R$ it follows that $f e=e$. Thus $e=f$ and hence $r f=r e=r$ so that $f$ is a right identity of $R$.

\section{References}

[1] Abrams G.D., "Rings with local units", Thesis (Ph.D.), University of Oregon, 1981, 82p.

[2] Abrams G.D., "Morita equivalence for rings with local units", Comm. Algebra 11 (1983), No. 8, 801-837.

[3] Abrams G.D., Ara P. and Molina M. S., Leavitt Path Algebras, Springer Lecture Notes, London, 2017.

[4] Ánh P.N. and Márki L., "Morita equivalence for rings without identity", Tsukuba J. Math. 11 (1987), No. 1, 1-16.

[5] Fuller K.R., "On rings whose left modules are direct sums of finitely generated modules", Proc. Amer. Math. Soc. 54 (1976), 39-44.

[6] Komatsu H., "The category of s-unital modules", Math. J. Okayma univ. 28 (1986), No. 1, 65-91.

[7] Lam T.Y., Exercises in classical ring theory, 2nd edition, Springer-Verlag, New York, 2003.

[8] Lundström P., "Separable Groupoid Rings", Comm. Algebra 34 (2006), 3029-3041.

[9] Mogami I. and Hongan M., "Note on commutativity of rings", Math. J. Okayama univ. 20 (1978), 21-24.

[10] Tominaga H., "On decompositions into simple rings", Math. J. Okayama univ. 17 (1975), No. 2, 159-163.

[11] Tominaga H., "On s-unital rings", Math. J. Okayama univ. 18 (1976), 117-134. 